\title{
Effectiveness of lumbar supports in low back functionality and disability in assembly-line workers
}

\author{
Ana Vanessa BATALLER-CERVERO ${ }^{1}$, Juan RABAL-PELAY ${ }^{\mathbf{1}}$, \\ Luis Enrique ROCHE-SERUENDO ${ }^{1}$, Belén LACÁRCEL-TEJERO ${ }^{2}$, \\ Andrés ALCÁZAR-CREVILLÉN ${ }^{2}$, Jose Antonio VILLALBA-RUETE ${ }^{3}$ \\ and Cristina CIMARRAS-OTAL ${ }^{1}$ * \\ ${ }^{1}$ Universidad San Jorge, Spain \\ ${ }^{2}$ Hospital Maz, Spain \\ ${ }^{3}$ BSH-Electrodomésticos España, Spain
}

Received September 11, 2018 and accepted December 27, 2018

Advanced Epub on J-STAGE January 16, 2019

\begin{abstract}
Low back pain (LBP) is a common problem in manufacturing workers. Several strategies have been proposed in order to reduce the pain and/or improve functionality. Among them, lumbar supports are a common solution prescribed for lumbar pain relief. Most of the studies in the literature only consider subjective sensations of the workers for evaluation assessment. This study applies biomechanical tests (a flexion-relaxation test and a functional movement evaluation test) to analyse the effectiveness of flexible lumbar supports in functionality and disability versus placebo intervention, consisting of kinesiotape placed on the low back without any stress. 28 workers participated in the study, randomised in control and intervention groups with a two months' intervention. None of the biomechanical tests showed statistical differences in between-groups pre-post changes. No benefits of wearing a flexible lumbar support during the workday have been found in these assemblyline workers versus placebo intervention.
\end{abstract}

Key words: Lumbar pain, Flexion-relaxation phenomenon, Flexible lumbar belt, Functionality, Manufacturing workers

\section{Introduction}

Musculoskeletal disorders (MSD) affect millions of European workers in all types of job and employment sectors every year, being the most prevalent occupational disease at European level. According to European ${ }^{1)}$ figures on recognized occupational diseases, MSD are the most com-

*To whom correspondence should be addressed. E-mail: ccimarras@usj.es

(C)2019 National Institute of Occupational Safety and Health mon occupational disease (39\% of the total). Among the different MSD, low back pain (LBP) is a recurrent common problem throughout the workers, with many sickness or absence episodes registered ${ }^{2}$. The highest incidence rate of musculoskeletal disorders occurs within the manufacturing sector: specifically, assembly-line workers of manufacturing companies who habitually stay most of the work day in a standing posture, making repetitive movements with upper limbs. Static positions, in addition to awkward lifting movements, could be mechanical causes for LBP in the workplace ${ }^{3,4)}$. Low back disorders show a 
higher incidence rate in manufacturing companies ${ }^{5)}$.

Several preventive strategies have been proposed for LBP prevention, such as ergonomic adjustments in the workplace; the use of declined surfaces ${ }^{6}$; fitness exercises; education on back mechanics and lifting techniques; and lumbar supports ${ }^{7}$ that can be used for LBP treatment as well as prevention ${ }^{8)}$.

The biomechanical effects of the lumbar support are the limitation of the flexo-extension and lateral bending movements ${ }^{9}$, the stabilisation of part of the spine and the decrease in the load on the trunk ${ }^{10)}$.

Systematic reviews have not found evidence for the effectiveness of lumbar supports for LBP prevention ${ }^{11,12)}$, but in these reviews only one study was performed on industry workers ${ }^{13)}$. Furthermore, most of the research found in the literature study the effectiveness of the lumbar supports in function of the pain incidence and number of days of sick leave. However, the studies that analyse biomechanical parameters are scant.

Several biomechanical assessment techniques are capable of identifying functional differences between LBP populations and healthy people ${ }^{14)}$. Among them, kinematics evaluations of functional movements have shown good accuracy in identifying patients with $\mathrm{LBP}^{15)}$.

Biomechanical analysis of everyday tasks, such as sitto-stand or lifting an object from the ground, can produce objective and reliable indexes about the patients' degree of functional impairment due to $\mathrm{LBP}^{16)}$. The motion patterns of LBP patients differ from those of healthy subjects. By measuring the vertical forces exerted and the relative positions of the back, an objective assessment of the LBP can be obtained ${ }^{16)}$.

Besides kinematic and dynamic measurements, surface electromyography (EMG) of low back muscles shows differences between LBP and healthy subjects during dynamic flexion tasks performed at peak flexion ${ }^{17)}$.

The flexion-relaxation phenomenon (FRP) represents a myoelectric silence of the low back extensor musculature during a standing to full flexion movement. The electrical signal silence in healthy people has been hypothesized to show the back extensor musculature being relieved of its supporting function by the passive tissues. This electrical signal reduction occurs in healthy subjects, but LBP patients fail in relaxing the erector spinae muscle and therefore, the FRP is not present. Persistent muscle activation restricts intervertebral motion in order to protect passive spinal structures from movements that cause pain ${ }^{18)}$. FRP is a valuable objective tool to aid in the diagnosis of $\mathrm{LBP}^{19)}$. The output of FRP in chronic LBP patients is dif- ferent from those with transient LBP. However, subjects who feel better due to an intervention, could relax the back extensor as the pain and function improve. It could be thought that as their muscle physiology returned to a healthier condition, the perceived pain and functionality will improve accordingly. It is proven that chronic LBP patients are not able to relax their spinal musculature due to a guarding behaviour or fear to movement. However, subjects who has a retrospective history of symptoms resolved show stronger relationship between changes in activation patterns during the test ${ }^{20)}$. Our subjects of study had suffered a shift leave due to this cause but they received treatment resolving their disorder.

The aim of this study is to investigate the effect of flexible lumbar support on back functionality and disability in assembly-line workers of a manufacturing company that suffered a previous episode of LBP in the last two years, compared to a placebo low back support made with kinesiotape without any stress, evaluated by means of biomechanical tests. FRP will be applied in order to check the disability of the workers and functional test will show the lumbar functionality degree. Both test will offer an objective evaluation of flexible lumbar support effectiveness. We hypothesise that workers with lumbar support will improve the functionality more than workers with placebo treatment.

\section{Subjects and Methods}

\section{Subjects}

The sample of the study was formed by 28 assemblyline workers of two plants of the same electrical appliances manufacturing company in Spain. Inclusion criteria were workers between 35 and $50 \mathrm{yr}$ old, having had a sick-leave due to lumbar pain in the last two years and working in an assembly-line in standing position during a full work day. Exclusion criteria were having a short-term contract with the company and presenting a lower than $90 \%$ punctuation in lumbar functionality test ${ }^{16)}$ in the previous intervention assessment. This threshold value has been calculated by means of a regression equation taking into account kinematic and kinetic variables of the functional movements. This value could detect with good sensitivity patients without low back functional impairment.

The recruitment of the participants of the study was done by the medical services of the company that had the information about the clinical history of all the workers. The subjects were randomly assigned to a control group (CG) or intervention group (IG). This randomisation was 
done by the researchers and communicated to the medical service responsible for the intervention. All the volunteers were informed about the aim of the study and the biomechanical tests, signing a written consent form.

\section{Ethical considerations}

All the procedures were conducted in accordance with the principles of the World Medical Association's Declaration of Helsinki. The study, all procedures and consent forms were approved by the ethical committee of the Universidad San Jorge.

\section{Measurement systems}

The methodology for the FRP was based on the work of Watson and colleagues ${ }^{21)}$. The protocol included 3D kinematic analysis of the low back by means of the motion capture system Smart-DX (BTS Bioengineering, Italy), composed by six $60 \mathrm{~Hz}$ infrared cameras, reflective markers and surface wireless EMG device, BTS FreeEMG 300 (BTS Bioengineering, Italy). The software provided by the system analysed synchronously kinematic and EMG of paraspinal muscles signals.

The recording sensor placement and setup is shown in Fig. 1a. For EMG, pre-gelled disposable silver chloride surface disk electrodes (2-cm diameter) were placed over the right and left longissimus and multifidus muscles at the level of L1-L2 and L4-L5, respectively. The skin underlying the electrode was cleaned with an alcohol pad to provide better conductivity. The EMG signal was captured with a sampling frequency of $1 \mathrm{kHz}$. The signal was filtered by a Hamming low pass filter of $350 \mathrm{~Hz}$ and a Hamming high pass filter of $20 \mathrm{~Hz}$. Seven passive reflective markers were adhered to the skin, located at D12$\mathrm{L} 1$ and S1 levels, in both greater trochanters and tips toe heart. ${ }^{22}$. Angle between S1 and L1 gives the true lumbar spine motion. Greater trochanters markers are used to see the pelvic movement and tips toe heart markers to detect the maximum flexion event.

The subject should stay at least $15 \mathrm{~s}$ in a static standing position to register initial myoelectric signals. After that, a lumbar flexion movement should be done for $10 \mathrm{~s}$, keeping the maximum lumbar flexion position for $1 \mathrm{~s}$ and a lumbar extension should be done for $10 \mathrm{~s}$, then repeating this movement three times. The rhythm of the movement is controlled by the laboratory operators by means of a time-counting signal, discarding the repetitions with a time lower or higher than this value.

The mean lumbar flexion velocity (FV), maximum flexion angle (MFA), elongation between L1 and L5 (El L1-

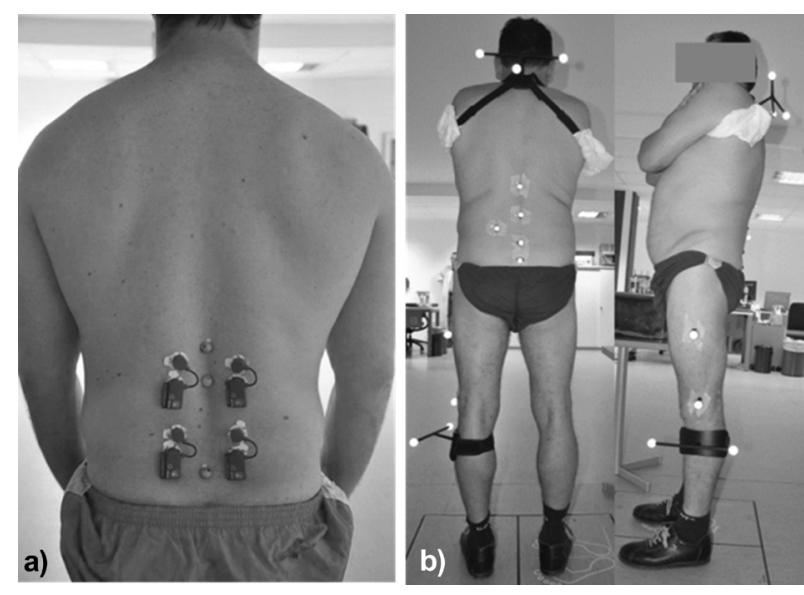

Fig. 1. a) EMG probes and reflective markers location for FRP. b) Reflective markers location for functional test in posterior frontal and sagittal planes.

L5), as well as the muscle activation previously, during and after the maximum lumbar flexion were registered. The beginning and the end of the movement phases were located by the operator by visually inspecting the lumbar flexion-extension variable. A flexion/relaxation ratio (FRR) of the activity during the forward flexion and fully flexed positions was calculated, dividing the maximal activity RMS during the flexion movement by the RMS during the fully flexed position. The combined discriminant validity of FRR for multifidus and longissimus in right and left sides offers a specificity of $75 \%$ and sensitivity of $93 \%{ }^{21}$. Higher ratios indicate relatively more flexion relaxation (less activation) of the muscles at full trunk flexion.

A second biomechanical evaluation was done to the subjects of the study including functional movements. They should perform a sit-to-stand task and lift three different weights (a box with handles designed for the test with $5 \mathrm{~kg}$ load, the same box empty and $10 \mathrm{~kg}$ load in this order) from a standing position, lifting the box up to the waist height and rotate the body to place the box on a table, following the protocol described by SánchezZuriaga and colleagues ${ }^{16)}$.

In this protocol, kinematic and dynamic analysis is implemented. Kinematic analysis was performed by means of a three-dimensional video photogrammetric system (Kinescan/IBV, Instituto de Biomecánica de Valencia, Valencia, Spain), which includes four cameras Pulnix TM-6740Cl with a resolution of $1,024 \times 768$ pixels and a frequency of $50 \mathrm{~Hz}$. The system also has two dynamometric platforms installed in parallel at ground level. The kinematic model represents the whole spine, the knee and hip motion. The model comprises 12 reflective markers of $25-\mathrm{mm}$ diameter, 
except on the thoracic and lumbar regions, where 15-mm markers were used (Fig. 1b). In order to avoid skin artefacts, markers on the $\mathrm{C} 7$ spinous process and leg were applied by means of rigid structures held in place by straps.

The protocol measures an index of normality for the sitto-stand task (SSI) and an index of normality for lifting a weight (LWI). These indexes are calculated on the basis of the force, velocity and acceleration values of flexion and extension phases of the movements, left and right asymmetry and repetitively of the movements. Moreover, a combined lumbar normality index (LNI) is offered by the system, as well as the collaboration index (CI), an indicator of the degree of collaboration of the subject in the assessment in order to show pretending patients. The data are compared to a normality database of healthy and pathological patients registers in order to calculate $\mathrm{LNI}^{23}$. A value lower than $90 \%$ in LNI could represent an altered low back functionality. The sensibility is $89 \%$ and the specificity presents a value of $100 \%$. CI is the result of the application of an algorithm comparing the subject data with pathological and pretending patients. A value lower than $50 \%$ indicates a non-collaborating patient. The sensibility and specificity of this index are $69 \%$ and $97 \%$ respectively ${ }^{24)}$.

\section{Experimental procedure}

A previous intervention evaluation of the workers was done, keeping several demographic and anthropometric variables and the current lumbar pain degree in a 5 points scale ( 0 no pain, 5 maximum pain). Besides this pain scale, the workers filled out the Nordic Musculoskeletal Questionnaire $^{25)}$.

The biomechanical tests of FRP and functional task evaluation were developed in a biomechanics laboratory by personnel external to the study, experts in clinical biomechanics.

The intervention period was two months long. Workers assigned to intervention group wore a lumbar support (Activemov Lombalift, BSN medical) eight hours during the work day. Medical services placed control group workers two strips of $10 \mathrm{~cm}$ in length kinesiotape in paraspinal muscles without stress every Monday of the intervention period and were replaced in case the strips detached (Fig. 2).

After two months, the same biomechanical evaluations were performed. The tests were done without lumbar supports or kinesiotape.

\section{Data analysis}

The variables were presented in number and percentage or mean and standard deviation if they were qualitative or
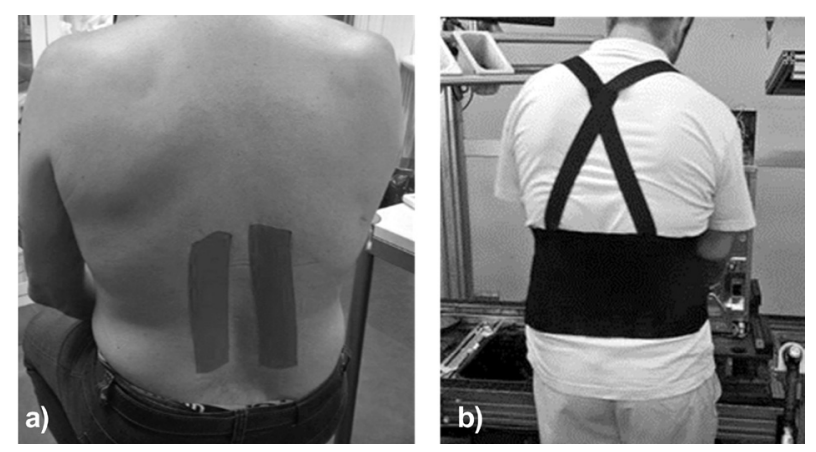

Fig. 2. a) Kinesiotape placement. b) lumbar support.

quantitative respectively. The normality of the variables was analysed applying the Shapiro-Wilk test.

A $t$-test was applied in order to calculate the differences between groups in baseline characteristics. The standardized differences or effect size (ES, 90\% confidence limits $[\mathrm{CL}]$ ) in the selected variables was calculated using the pooled standardized difference. Threshold values for Cohen's ES statistics were $>0.2$ (small), $>0.6$ (moderate), and

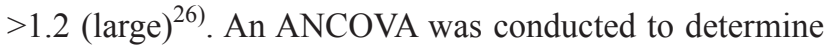
the between-group differences using the pre-test as a covariate.

IBM SPSS statistics version 21 was used for all statistical analyses. A value of $p$ lower than 0.05 was considered statistically significant.

\section{Results}

A total of 28 workers participated in the study (22 men and 6 women) with a mean age of $43 \mathrm{yr}$. All the subjects had a score equal to or higher than $90 \%$ of LNI in the functional test. The number of workers assigned to intervention and control groups were 14. Baseline characteristics of the participants are given in Table 1. No differences between control and intervention groups were found at baseline in age, weight, height and BMI variables. The degree of lumbar pain does not show differences between groups. All the subjects showed lumbar pain in the last 12 months according to the Nordic Questionnaire.

\section{Within-group changes}

Within-group comparison between pre and post evaluations is shown in Table 2. Kinematic variables of maximum flexion angle, elongation and flexion velocity and $\mathrm{F} /$ $\mathrm{R}$ ratios for right and left sides longissimus and multifidus of the Flexion-Relaxation test are shown in Table 2 together with lumbar normality index, collaboration index, 
Table 1. Baseline characteristics of the sample

\begin{tabular}{lcc}
\hline & Intervention group $(\mathrm{n}=14)$ & Control group $(\mathrm{n}=14)$ \\
\hline Age (yr), Mean (SD) & $43(7)$ & $41(7)$ \\
Sex, N (\%) & & \\
$\quad$ Male & $11(78.5)$ & $11(78.5)$ \\
$\quad$ Female & $3(21.5)$ & $3(21.5)$ \\
Height (cm), Mean (SD) & $172(10.0)$ & $173(10.1)$ \\
Weight (kg), Mean (SD) & $79.1(13.5)$ & $82.2(20.0)$ \\
BMI, Mean (SD) & $26.4(2.8)$ & $23.3(6.1)$ \\
Lumbar pain, Mean (SD) & $3.6(0.8)$ & $2.7(1.7)$ \\
12 months LBP, N (\%) & $100(0)$ & $100(0)$ \\
\hline
\end{tabular}

BMI: Body Mass Index; LBP: Low Back Pain.

sit-to-stand normality index and lifting a weight index.

There were found differences between pre and post evaluation in longissimus FR ratio for right and left sides in intervention group and in left side longissimus FR ratio in control group. The effect size is moderate for the three variables $(>0.6)$. The rest of the variables shown a trivial or small effect size.

\section{Between-group changes}

Analysing the change in the variables between groups no significant differences have been found in any of the variables. The effect size is trivial in most of the variables. Only a moderate value has been found in multifidus flexrelax ratio in right side (Table 3 ).

\section{Discussion}

The aim of the present study was investigating the effectiveness of flexible lumbar supports on functionality and disability analysed by means of biomechanical tests. Our results do not show evidence to support the benefits of lumbar supports for low back pain or lumbar functionality versus placebo intervention in assembly line workers with a previous episode of lumbar shift-leave in the past two years. Intervention group and control group do not show differences in the low back pain level at the beginning of the intervention period. Within-group comparison shows differences between pre and post evaluation in right and left longissimus flex-relax ratios for intervention group with a moderate effect size. An increase in the ratios are found that could be interpret as a higher relaxation of the spinal muscles in the flexed position. In betweengroup comparison of pre-post evaluations changes in the functional movements test and flexion-relaxation test, no statistical differences were found and the effect size of the comparison were trivial in all the variables.
Although it can be seen an improvement in two of the FR ratios in the disability test, a significant change in the rest of the variables has not been found. Moreover, control group also shown a better value of longissimus FR ratio in post evaluation. In spite of that, the between-group pre-post changes comparison does not show significant difference, concluding that there are not evidences to support that the intervention with flexible lumbar support could have beneficial effects on lumbar disability or functionality. The improvement in LL-FR, found in both intervention and control group may be due to the placebo effect in people that previously have experienced a leave for LBP and that could present guarding behaviours at time to develop the test. This guarding behaviour could have been controlled thanks to an intervention of lumbar support or placebo. Besides this change in FRR, the rest of the variables in disability test and the functionality didn't show an improvement in post evaluation. There has not been found any study in the literature that makes use of these technologies in the evaluation of the effectiveness of lumbar supports.

Lumbar spinal orthoses have achieved considerable fame in many self-reported survey studies, in which people have claimed that using these orthoses helped them continue their daily activities with considerable minimal discomfort or even reduced their pain level. The perception of people wearing lumbar supports is that they feel more stable and safer while they are doing physical work. Lumbar supports may restrict lumbar motion, preventing the loading of certain spine structures ${ }^{9)}$. Despite the subjective perceptions of the lumbar supports reported in previous studies, these studies do not show any evidence of positive effects according to biomechanical measurements.

The ergonomics journals are plenty of publications of studies that analyse the effects of lumbar supports for the prevention or treatment of LPB in workplace. However, when comparing the effectiveness of lumbar supports in the 
Table 2. Mean (SD) of biomechanical test variables in control and intervention groups in pre and post and effect size of within-group change (with $90 \%$ confidence interval)

\begin{tabular}{|c|c|c|c|c|c|c|}
\hline & \multicolumn{3}{|c|}{ Intervention group $(\mathrm{n}=14)$} & \multicolumn{3}{|c|}{ Control group $(n=14)$} \\
\hline & Pre & Post & ES (CL90\%) & Pre & Post & ES (CL90\%) \\
\hline MFA & $75.3(8.2)$ & $73.6(9.2)$ & $-0.2(-0.8 ; 0.4)$ & $77.5(9.9)$ & $74.6(10.4)$ & $-0.3(-0.9 ; 0.3)$ \\
\hline El L1-L5 & $64.3(13.9)$ & $65.6(13.1)$ & $0.1(-0.5 ; 0.7)$ & $69.0(12.9)$ & $67.7(12.5)$ & $-0.1(-0.7 ; 0.5)$ \\
\hline $\mathrm{FV}(\%)$ & $22.7(4.1)$ & $24.3(8.2)$ & $0.2(-0.4 ; 0.8)$ & $25.8(3.9)$ & $27.1(8.0)$ & $0.2(-0.4 ; 0.8)$ \\
\hline LL-F/R & $1.3(0.4)$ & $1.9(0.7)^{*}$ & $1.0(0.3 ; 1.7)$ & $1.1(0.6)$ & $1.7(0.8)^{*}$ & $0.8(0.1 ; 1.4)$ \\
\hline LR-F/R & $1.9(0.5)$ & $2.6(1.0)^{*}$ & $0.8(0.1 ; 1.3)$ & $1.6(0.8)$ & $2.2(1.3)$ & $0.5(-0.0 ; 1.1)$ \\
\hline $\mathrm{ML}-\mathrm{F} / \mathrm{R}$ & $2.8(1.3)$ & $3.0(2.3)$ & $0.1(-0.5 ; 0.7)$ & $1.9(1.0)$ & $2.4(1.5)$ & $0.3(-0.2 ; 1)$ \\
\hline MR-F/R & $3.0(1.4)$ & $2.5(1.2)$ & $-0.3(-1.0 ; 0.2)$ & $2.3(1.4)$ & $2.8(2.3)$ & $0.2(-0.3 ; 0.8)$ \\
\hline LNI & $96.2(2.5)$ & $96.9(2.4)$ & $0.2(-0.3 ; 0.9)$ & $97.1(2.7)$ & $98.0(1.6)$ & $0.4(-0.2 ; 1)$ \\
\hline $\mathrm{CI}$ & $94.0(4.8)$ & $92.8(6.5)$ & $-0.2(-0.8 ; 0.4)$ & $95.0(4.9)$ & $96.5(4.3)$ & $0.3(-0.3 ; 0.9)$ \\
\hline SSI & $95.5(3.2)$ & $96.0(3.0)$ & $0.1(-0.4 ; 0.8)$ & $97.0(3.4)$ & $97.7(2.1)$ & $0.2(-3 ; 0.8)$ \\
\hline WI & $98.3(2.6)$ & $98.5(1.3)$ & $0.1(-0.5 ; 0.7)$ & $97.8(2.5)$ & $98.7(1.5)$ & $0.4(-0.2 ; 1.5)$ \\
\hline
\end{tabular}

${ }^{*} p<0.05$ in the statistical test between pre and post evaluation. CL: Confidence limit.

MFA: maximum flexion angle; El L1-L5: elongation between L1 and L5; FV: forward velocity; LL-F/R and LR-F/R: left and right longissimus flex-relax ratio respectively; ML-F/R and MR-F/R: left and right multifidus flex-relax ratio respectively; LNI: lumbar normality index; CI: collaboration index; SSI: sit-to-stand normality index; LWI: lifting weight normality index; ES: effect size.

Table 3. Between-group pre-post mean differences and effect size (ES) (with $90 \%$ confidence interval)

\begin{tabular}{|c|c|c|c|}
\hline & Intervention group $(\mathrm{n}=14)$ & Control group $(n=14)$ & Intervention-control \\
\hline & Pre-Post & Pre-Post & ES (CL90\%) \\
\hline MFA & 1.7 & 2.9 & $0.1(-0.7 ; 0.4)$ \\
\hline El L1-L5 & -1.3 & 1.3 & $-0.1(-0.8 ; 04)$ \\
\hline $\mathrm{FV}(\% / \mathrm{s})$ & -1.6 & -1.3 & $-0.0(-0.6 ; 0.5)$ \\
\hline LL-F/R & -0.6 & -0.6 & $0.0(-0.6 ; 0.6)$ \\
\hline LR-F/R & -0.7 & -0.6 & $-0.1(-0.7 ; 0.4)$ \\
\hline ML-F/R & -0.2 & -0.5 & $0.2(-0.3 ; 0.8)$ \\
\hline MR-F/R & 0.5 & -0.5 & $0.6(0.0 ; 1.3)$ \\
\hline LNI & -0.7 & 0.9 & $0.0(-0.5 ; 0.7)$ \\
\hline $\mathrm{CI}$ & 1.2 & -1.5 & $0.5(-0.0 ; 1.1)$ \\
\hline SSI & -0.5 & -0.7 & $0.0(-0.5 ; 0.6)$ \\
\hline LWI & -0.2 & -0.9 & $0.2(-0.3 ; 0.8)$ \\
\hline
\end{tabular}

MFA: maximum flexion angle; E1 L1-L5: elongation between L1 and L5; FV: forward velocity; LL-F/R and LR-F/R: left and right longissimus flex-relax ratio respectively; ML-F/R and MR-F/R: left and right multifidus flex-relax ratio respectively; LNI: lumbar normality index; CI: collaboration index; SSI: sit-to-stand normality index; LWI: lifting a weight normality index; ES: effect size.

scientific literature, there is a lack of homogeneity in terms of the type of lumbar support used and the outcome measurements, offering contradictory conclusions. Many of the studies published offer low methodological quality, as has been concluded in different systematic reviews $8,11,12,27)$. A control of the compliance of the treatment, the type of lumbar support and patients and outcomes measured should be standardised in order to compare the studies.

Among the previously published studies that include randomised trials, the study completed by Morrissette and colleagues $^{28)}$ finds that the use of inextensible lumbar supports is more effective in reducing disability level caused by low back pain than standard care alone, concluding that inextensible lumbar supports improve self-reported function of the patients. Other randomised trial concludes that lumbar belts are effective in subacute low back pain by reducing pain level and functionality ${ }^{29,30)}$. Although these studies prove the effectiveness of lumbar support, the methods employed are dependent on the patient's subjective sensation and not based on objective biomechanical 
measurements. The discrepancies with our results could be due to the fact that the subjective sensation of the subjects wearing a lumbar support is a sensation of comfort, or even of pain relief, even though no biomechanical improvements were produced. Furthermore, the type of lumbar support analysed in these studies could be different than that used in our study.

In line with our results, a review does not find a conclusive evidence to support lumbar belt use to prevent or reduce lost time from occupational $\mathrm{LBP}^{8)}$. Other studies neither found reduction in pain nor disability with the use of rigid or semi-flexible corsets ${ }^{31)}$. Prospective studies, such as those of Wassel and colleagues ${ }^{32)}$, do not show any association between the use of lumbar support and the reduction of the incidence of LBP.

Although no benefits in lumbar functionality are found in our study in workers wearing a flexible lumbar support, we also did not see a deterioration of spine function with the biomechanical test applied. It is thought that the longterm use of a lumbar orthosis could generate a weakening of the trunk muscles. The results in the literature are contradictory and do not seem to confirm this approach. A systematic review and meta-analysis of studies shows that more research is needed to support the hypothesis that lumbar supports decrease the back muscle force ${ }^{9}$. Other studies, such as that of Kawchuf and colleagues ${ }^{33)}$, suggest that the use of lumbar supports do not generate a deterioration of spine function.

\section{Limitations}

There are several limitations of our study. First of all, the number of workers that wanted to participate in the study was small. Secondly, in this study, biomechanical tests were applied to assess the functionality and disability of workers wearing a lumbar support versus workers with placebo intervention. Although these methods provide an objective measurement of effectiveness of lumbar supports, the subjective appreciation of the workers has not been taken into consideration as a study variable. The level of low back pain disability measured by a validated questionnaire, such as Oswestry or Rolland Morris ${ }^{34)}$, has not been registered before and after the intervention period. This could show information about the level of disability perceived by the workers. Moreover, the lumbar pain scale could have also supported the discomfort experienced by the subjects in post-intervention evaluation.

Finally, although the kinesiotape as placebo was placed each intervention week, compliance with wearing the lumbar support was not registered by the medical service. In a future study, a better control of this point will be considered.

\section{Conclusions}

Our data found no improvement in lumbar functionality and disability for assembly-line workers with previous episodes of sick leave by LBP when wearing a flexible lumbar support versus a worker with a placebo intervention.

\section{Acknowledgement}

This research has been done thanks to the support of the Cátedra "Empresa Sana" signed between BSH/E, MAZ and Universidad San Jorge and has been supported by Operative Program ERDF Aragon 2014-2020, "Building Europe from Aragon", Research Group ValorA.

\section{References}

1) European Commission (2000) European Occupational Diseases Statistics (EODS)-Phase 1 methodology. Eurostat working papers. Population and social conditions 3/2000/E/No 19. Reference metadata on Occupational Diseases, Compiling Agency: Statistical Office of the European Communities (Eurostat), Luxemburg.

2) Wynne-Jones G, Cowen J, Jordan JL, Uthman O, Main CJ, Glozier N, van der Windt D (2014) Absence from work and return to work in people with back pain: a systematic review and meta-analysis. Occup Environ Med 71, 448-56. [Medline] [CrossRef]

3) Sterud T, Tynes T (2013) Work-related psychosocial and mechanical risk factors for low back pain: a 3-year followup study of the general working population in Norway. Occup Environ Med 70, 296-302. [Medline] [CrossRef]

4) Driscoll T, Jacklyn G, Orchard J, Passmore E, Vos T, Freedman G, Lim S, Punnett L (2014) The global burden of occupationally related low back pain: estimates from the Global Burden of Disease 2010 study. Ann Rheum Dis 73, 975-81. [Medline] [CrossRef]

5) Bataller-Cervero AV, Cimarras-Otal C, Sanz-López F, Lacárcel-Tejero B, Alcázar-Crevillén A, Villalba Ruete JA (2016) Musculoskeletal disorders assessment using sickleaves registers in a manufacturing plant in Spain. Int J Ind Ergon 56, 124-9. [CrossRef]

6) Gallagher KM, Callaghan JP (2016) Standing on a declining surface reduces transient prolonged standing induced low back pain development. Appl Ergon 56, 76-83. [Medline] [CrossRef]

7) Lahad A, Malter AD, Berg AO, Deyo RA (1994) The effectiveness of four interventions for the prevention of low back pain. JAMA 272, 1286-91. [Medline] [CrossRef]

8) Ammendolia C, Kerr MS, Bombardier C (2005) Back belt use for prevention of occupational low back pain: a 
systematic review. J Manipulative Physiol Ther 28, 128-34. [Medline] [CrossRef]

9) van Poppel MN, de Looze MP, Koes BW, Smid T, Bouter LM (2000) Mechanisms of action of lumbar supports: a systematic review. Spine 25, 2103-13. [Medline] [CrossRef]

10) Nachemson A, Schultz A, Andersson G (1983) Mechanical effectiveness studies of lumbar spine orthoses. Scand J Rehabil Med Suppl 9, 139-49. [Medline]

11) Jellema $P$, van Tulder MW, van Poppel MN, Nachemson AL, Bouter LM (2001) Lumbar supports for prevention and treatment of low back pain: a systematic review within the framework of the Cochrane Back Review Group. Spine 26, 377-86. [Medline] [CrossRef]

12) van Poppel MN, Hooftman WE, Koes BW (2004) An update of a systematic review of controlled clinical trials on the primary prevention of back pain at the workplace. Occup Med (Lond) 54, 345-52. [Medline] [CrossRef]

13) van Poppel MN, Koes BW, van der Ploeg T, Smid T, Bouter LM (1998) Lumbar supports and education for the prevention of low back pain in industry: a randomized controlled trial. JAMA 279, 1789-94. [Medline] [CrossRef]

14) Lehman GJ (2004) Biomechanical assessments of lumbar spinal function. How low back pain sufferers differ from normals. Implications for outcome measures research. Part I: kinematic assessments of lumbar function. J Manipulative Physiol Ther 27, 57-62. [Medline] [CrossRef]

15) Bishop JB, Szpalski M, Ananthraman SK, McIntyre DR, Pope MH (1997) Classification of low back pain from dynamic motion characteristics using an artificial neural network. Spine 22, 2991-8. [Medline] [CrossRef]

16) Sánchez-Zuriaga D, López-Pascual J, Garrido-Jaén D, de Moya MF, Prat-Pastor J (2011) Reliability and validity of a new objective tool for low back pain functional assessment. Spine 36, 1279-88. [Medline] [CrossRef]

17) Mannion AF, Taimela S, Müntener M, Dvorak J (2001) Active therapy for chronic low back pain part 1. Effects on back muscle activation, fatigability, and strength. Spine 26, 897-908. [Medline] [CrossRef]

18) Kaigle AM, Wessberg P, Hansson TH (1998) Muscular and kinematic behavior of the lumbar spine during flexion-extension. J Spinal Disord 11, 163-74. [Medline] [CrossRef]

19) Colloca CJ, Hinrichs RN (2005) The biomechanical and clinical significance of the lumbar erector spinae flexion-relaxation phenomenon: a review of literature. J Manipulative Physiol Ther 28, 623-31. [Medline] [CrossRef]

20) McGorry RW, Lin JH (2012) Flexion relaxation and its relation to pain and function over the duration of a back pain episode. PLoS One 7, e39207. [Medline] [CrossRef]

21) Watson PJ, Booker CK, Main CJ, Chen AC (1997) Surface electromyography in the identification of chronic low back pain patients: the development of the flexion relaxation ratio. Clin Biomech (Bristol, Avon) 12, 165-71. [Medline]
[CrossRef]

22) Biviá-Roig G, Lisón JF, Sánchez-Zuriaga D (2019) Effects of pregnancy on lumbar motion patterns and muscle responses. Spine J 19, 364-71. [Medline] [CrossRef]

23) López-Pascual J, Peydro-De-Moya MF, Garrido-Jaén JD, Bausá-Peris R, Villadeamigo-Panchón MJ (2017) Analyse of the use of functional validation tools for work related lumbar pain. Rehabilitacion 51, 16-23 (in Spanish).

24) Peydro MF, Bausa R, Barberá R, Cortés A, Sanchez J, Durá JV, Prat J, Ramiro J (2004) Biomechanics quantification of the collaboration degree of patients with low back pain. IV Congreso Nacional de la SETLA (in Spanish).

25) Kuorinka I, Jonsson B, Kilbom A, Vinterberg H, BieringSørensen F, Andersson G, Jørgensen K (1987) Standardised Nordic questionnaires for the analysis of musculoskeletal symptoms. Appl Ergon 18, 233-7. [Medline] [CrossRef]

26) Hopkins WG, Marshall SW, Batterham AM, Hanin J (2009) Progressive statistics for studies in sports medicine and exercise science. Med Sci Sports Exerc 41, 3-13. [Medline] [CrossRef]

27) van Duijvenbode IC, Jellema P, van Poppel MN, van Tulder MW (2008) Lumbar supports for prevention and treatment of low back pain. Cochrane Database Syst Rev CD001823. [Medline]

28) Morrisette DC, Cholewicki J, Logan S, Seif G, McGowan S (2014) A randomized clinical trial comparing extensible and inextensible lumbosacral orthoses and standard care alone in the management of lower back pain. Spine 39, 1733-42. [Medline] [CrossRef]

29) Calmels P, Queneau P, Hamonet C, Le Pen C, Maurel F, Lerouvreur C, Thoumie P (2009) Effectiveness of a lumbar belt in subacute low back pain: an open, multicentric, and randomized clinical study. Spine 34, 215-20. [Medline] [CrossRef]

30) Gibson JNA, Ahmed M (2002) The effectiveness of flexible and rigid supports in patients with lumbar backache. $\mathrm{J}$ Orthop Med 24, 86-9. [CrossRef]

31) Million R, Nilsen KH, Jayson MI, Baker RD (1981) Evaluation of low back pain and assessment of lumbar corsets with and without back supports. Ann Rheum Dis 40, 449-54. [Medline] [CrossRef]

32) Wassell JT, Gardner LI, Landsittel DP, Johnston JJ, Johnston JM (2000) A prospective study of back belts for prevention of back pain and injury. JAMA 284, 2727-32. [Medline] [CrossRef]

33) Kawchuk GN, Edgecombe TL, Wong AY, Cojocaru A, Prasad N (2015) A non-randomized clinical trial to assess the impact of nonrigid, inelastic corsets on spine function in low back pain participants and asymptomatic controls. Spine J 15, 2222-7. [Medline] [CrossRef]

34) Koç M, Bayar B, Bayar K (2018) A comparison of back pain functional scale with Roland Morris Disability Questionnaire, Oswestry Disability Index, and Short Form 36-Health Survey. Spine 43, 877-82. [Medline] [CrossRef] 